S. Sun, G. R. MacCartney Jr., and T. S. Rappaport, "A novel millimeter-wave channel simulator and applications for 5G wireless communications," 2017 IEEE International Conference on Communications (ICC), Paris, May 2017.

\title{
A Novel Millimeter-Wave Channel Simulator and Applications for 5G Wireless Communications
}

\author{
Shu Sun, George R. MacCartney Jr., and Theodore S. Rappaport \\ NYU WIRELESS and NYU Tandon School of Engineering, New York University, Brooklyn, NY, USA 11201 \\ $\{$ ss7152,gmac,tsr\}@nyu.edu
}

\begin{abstract}
This paper presents details and applications of a novel channel simulation software named NYUSIM, which can be used to generate realistic temporal and spatial channel responses to support realistic physical- and link-layer simulations and design for fifth-generation (5G) cellular communications. NYUSIM is built upon the statistical spatial channel model for broadband millimeter-wave (mmWave) wireless communication systems developed by researchers at New York University (NYU). The simulator is applicable for a wide range of carrier frequencies $(500 \mathrm{MHz}$ to $100 \mathrm{GHz})$, radio frequency (RF) bandwidths $(0$ to $800 \mathrm{MHz})$, antenna beamwidths $\left(7^{\circ}\right.$ to $360^{\circ}$ for azimuth and $7^{\circ}$ to $45^{\circ}$ for elevation), and operating scenarios (urban microcell, urban macrocell, and rural macrocell), and also incorporates multiple-input multiple-output (MIMO) antenna arrays at the transmitter and receiver. This paper also provides examples to demonstrate how to use NYUSIM for analyzing MIMO channel conditions and spectral efficiencies, which show that NYUSIM is an alternative and more realistic channel model compared to the 3rd Generation Partnership Project (3GPP) and other channel models for mmWave bands.
\end{abstract}

Index Terms-5G, mmWave, simulator, NYUSIM, MIMO.

\section{INTRODUCTION}

The construction and implementation of channel models are becoming increasingly important for wireless communication system design, and computer-aided design tools such as channel simulators are essential for performance evaluation of communications systems and for simulating network deployments, before moving forward with new technologies.

There are several channel simulators that have been developed and used by previous researchers [1]-[7]. For instance, Smith [8] built simulation software for indoor and outdoor propagation channels by making use of the two-ray Rayleigh fading channel model developed by Clarke [9]. Fraunhofer Heinrich Hertz Institute developed a 3-D multi-cell channel model that can accurately predict the performance for an urban macrocell setup with commercial high-gain antennas, upon which a channel simulator has been built that supports features such as time evolution, scenario transitions, and so on [2]. A channel simulator for indoor scenarios was developed for machine-to-machine applications [3]. Rappaport and Seidel developed a measurement-based statistical indoor channel model named SIRCIM (Simulation of Indoor Radio Channel Impulse Response Models) for the early development of WiFi [4] and the corresponding simulation software to generate channel impulse responses (CIRs) for indoor channels operating from $10 \mathrm{MHz}$ to $60 \mathrm{GHz}$. A similar open-source RF propagation simulator is SMRCIM (Simulation of Mobile

Sponsorship for this work was provided by the NYU WIRELESS Industrial Affiliates program and NSF research grants 1320472, 1302336, and 1555332.
Radio Channel Impulse Response Models), that was useful for simulating outdoor channels [5], [6]. Another software simulation program, called BERSIM [7], developed by Fung et al., was able to simulate mobile radio communication links and calculate average bit error rate (BER) and bit-by-bit error patterns, that was useful for evaluating link quality in real time without requiring any radio frequency hardware.

In this paper, we introduce an open-source channel simulator named NYUSIM [10], which has been developed based on extensive real-world wideband propagation channel measurements at multiple millimeter-wave (mmWave) frequencies from 28 to $73 \mathrm{GHz}$ in various outdoor environments in urban microcell (UMi), urban macrocell (UMa), and rural macrocell (RMa) environments [11]-[19]. NYUSIM provides an accurate rendering of actual channel impulse responses in both time and space, as well as realistic signal levels that were measured, and is applicable for a wide range of carrier frequencies from 500 $\mathrm{MHz}$ to $100 \mathrm{GHz}$, and RF bandwidths from 0 to $800 \mathrm{MHz}$. As of early 2017, over 7,000 downloads of NYUSIM have been recorded. The source code was written in MATLAB [20] and a platform-independent graphical user interface (GUI) was created to facilitate the use of NYUSIM on machines using either Windows or Macintosh operating systems even without MATLAB installed.

\section{Channel Model Supported By NYUSIM}

Omnidirectional channel models have widely been studied and adopted by industry and researchers around the world to assist in wireless system design, yet directional channel models are also important to properly design and implement antenna arrays to exploit spatial diversity and/or beamforming gain in multiple-input multiple-output (MIMO) systems [21], [22]. NYUSIM generates sample functions of the temporal and spatial CIRs from both omnidirectional and directional channel models that are borne out by measurements and models of NYU WIRELESS [10]-[19]. This section provides a brief overview of the path loss model and clustering algorithm used in NYUSIM.

\section{A. Path Loss Model}

The close-in free space reference distance (CI) path loss model with a $1 \mathrm{~m}$ reference distance [12], [15], [17], [19], and an extra attenuation term due to various atmospheric conditions [23], is employed in NYUSIM, which is expressed as [12], [15], [24]:

$$
\begin{aligned}
\mathrm{PL}^{\mathrm{CI}}(f, d)[\mathrm{dB}]= & \operatorname{FSPL}(f, 1 \mathrm{~m})[\mathrm{dB}]+10 n \log _{10}(d) \\
& +\operatorname{AT}[\mathrm{dB}]+\chi_{\sigma}^{\mathrm{CI}}, \\
& \text { where } d \geq 1 \mathrm{~m}
\end{aligned}
$$



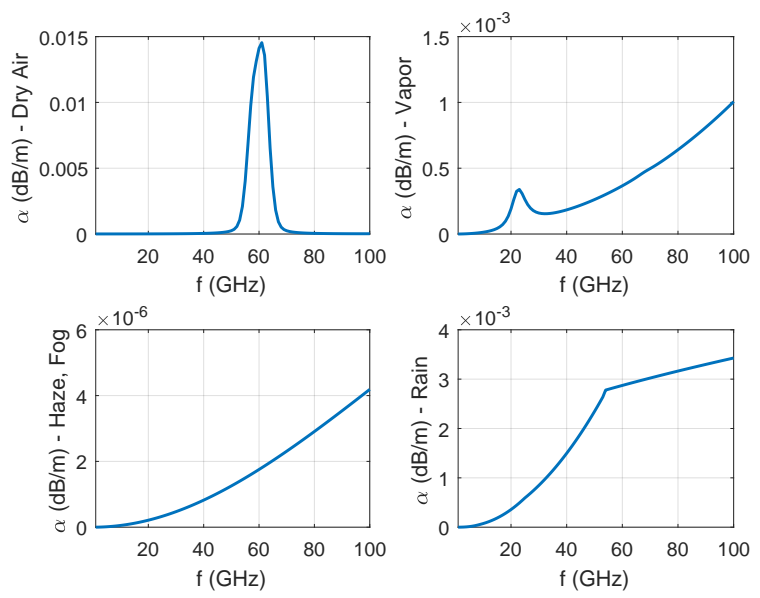

Fig. 1: Propagation attenuation due to dry air, vapor, haze, and rain at mmWave frequencies, with a barometric pressure of $1013.25 \mathrm{mbar}$, a relative humidity of $80 \%$, a temperature of $20^{\circ} \mathrm{C}$, and a rain rate of $5 \mathrm{~mm} / \mathrm{hr}[23]$.

where $f$ denotes the carrier frequency in $\mathrm{GHz}, d$ is the $3 \mathrm{D}$ T-R separation distance, $n$ represents the path loss exponent (PLE), AT is the attenuation term induced by the atmosphere, $\chi_{\sigma}^{\mathrm{CI}}$ is a zero-mean Gaussian random variable with a standard deviation $\sigma$ in $\mathrm{dB}$, and $\operatorname{FSPL}(f, 1 \mathrm{~m})$ denotes the free space path loss in $\mathrm{dB}$ at a T-R separation distance of $1 \mathrm{~m}$ at the carrier frequency $f$ :

$$
\begin{aligned}
\operatorname{FSPL}(f, 1 \mathrm{~m})[\mathrm{dB}] & =20 \log _{10}\left(\frac{4 \pi f \times 10^{9}}{c}\right) \\
& =32.4[\mathrm{~dB}]+20 \log _{10}(f)
\end{aligned}
$$

where $c$ is the speed of light in a vacuum, and $f$ is in $\mathrm{GHz}$. The term AT is characterized by:

$$
\mathrm{AT}[\mathrm{dB}]=\alpha[\mathrm{dB} / \mathrm{m}] \times d[\mathrm{~m}]
$$

where $\alpha$ is the attenuation factor in $\mathrm{dB} / \mathrm{m}$ for the frequency range of $1 \mathrm{GHz}$ to $100 \mathrm{GHz}$, which includes the collective attenuation effects of dry air (including oxygen), water vapor, rain, and haze [23]. The parameter $d$ is the 3D T-R separation distance in (1).

Fig. 11illustrates examples of propagation attenuation values due to dry air, vapor, haze, and rain at mmWave frequencies from $1 \mathrm{GHz}$ to $100 \mathrm{GHz}$, with a barometric pressure of 1013.25 mbar, a relative humidity of $80 \%$, a temperature of $20^{\circ} \mathrm{C}$, and a rain rate of $5 \mathrm{~mm} / \mathrm{hr}$. These results were obtained and reproduced from five reported controlled experiments on atmospheric attenuation [23].

Note that the CI model inherently has an intrinsic frequency dependency of path loss already embedded within the FSPL term, making it useful from below $1 \mathrm{GHz}$ to above 100 $\mathrm{GHz}$ [12], [15], [17], [19]. A useful property of (1) is that $10 n$ describes path loss in $\mathrm{dB}$ in terms of decades of distances beginning at $1 \mathrm{~m}$ (making it very easy to compute power over distance in one's mind). The CI path loss model is based on fundamental principles of wireless propagation, dating back to Friis and Bullington, where the PLE parameter offers insight into path loss based on the environment, having a PLE value of 2 in free space (as shown by Friis) and a value of 4 for the asymptotic two-ray ground bounce propagation model (as shown by Bullington [25], [26]). Hata adopted these fundamentals in his famous path loss model for the very early days of cellular [27], [28]. Standardizing to a reference distance of $1 \mathrm{~m}$ makes comparisons of measurements and models simple, and provides a universal definition for the PLE, while enabling intuition and rapid computation of path loss [15]. Compared with the existing alpha-beta-gamma (ABG) path loss model used in 3GPP/ITU channel models [15], [17], the CI model uses fewer parameters while offering intuitive physical appeal, better model parameter stability, and better prediction performance over a vast range of microwave and mmWave frequencies, distances, and scenarios, with fewer parameters [15].

In Version 1.4 of NYUSIM [10], the PLE $n$ is set to the free space PLE of 2 with a shadow fading standard deviation of 4.0 $\mathrm{dB}$ for the line-of-sight (LOS) environment, while the PLE and shadow fading standard deviation are respectively set to 3.2 and $7.0 \mathrm{~dB}$ for the non-LOS (NLOS) environment [12], [13], for both UMi and UMa scenarios. For the RMa scenario, the LOS PLE and shadow fading standard deviation are 2.16 and 4.0 dB, respectively, while the NLOS PLE and shadow fading standard deviation are 2.75 and $8.0 \mathrm{~dB}$, respectively [18], [29]. All parameters may be adjusted by the user in MATLAB, as source code is provided using an open license [10].

\section{B. Wideband Temporal/Spatial Clustering Algorithm}

The statistical spatial channel model (SSCM) [13] in NYUSIM utilizes time clusters (TC) and spatial lobes (SL) to model the omnidirectional CIR and corresponding joint angle of departure (AOD)/angle of arrival (AOA) power spectra, which have been used successfully in modeling mmWave channels [13]. Time clusters are composed of multipath components (MPCs) traveling closely in time, and that arrive from potentially different angular directions in a short excess delay time window. Spatial lobes represent main directions of arrival (or departure) where energy arrives over several hundreds of nanoseconds. This SSCM structure is motivated by field measurements, which have shown that multiple paths within a TC can arrive at unique pointing angles [13], detectable due to high gain directional antennas, and this feature has not been modeled in current 3GPP and WINNER models [30], [31]. The TCSL approach implements a physically-based clustering scheme (e.g., the use of a fixed inter-cluster void interval representing the minimum propagation time between likely reflection or scattering objects) derived from field observations, and can be used to extract TC and SL statistics for any raytracing or measurement data sets [13]. The time-partitioning methodology delineates the beginning and end times of each time cluster, using a 25 ns minimum inter-cluster void interval. Sequentially arriving MPCs that occur within $25 \mathrm{~ns}$ of each other are assumed to belong to one TC. This is different than joint time-angle distributions [21].

It is worth noting that in 3GPP TR 38.900 Release 14 for above $6 \mathrm{GHz}$ [32], the number of clusters is unrealistically large. For example, in the UMi street canyon scenario, the number of clusters in the LOS environment is as high as 12, and 19 in the NLOS environment, which is not supported by the real-world measurements at mmWave bands [11]-[14]. In 
contrast, in the SSCM upon which NYUSIM is based [13], the number of time clusters ranges from 1 to 6 , and the mean number of spatial lobes is about 2 and is upper-bounded by 5 , which are obtained from field observations and are much smaller than those in the 3GPP channel model [32]. The impractically large number of clusters in the 3GPP channel model causes a higher rank of simulated mmWave channels, and unrealistic eigen-channel distributions, thereby yielding inaccurate spectral efficiency prediction for mmWave channels.

\section{GRAPHICAL USER INTERFACE AND SiMUlatoR BASICS}

The screenshot in Fig. 2 shows the graphical user interface (GUI) of NYUSIM. The simulator performs Monte Carlo simulations, generating certain numbers of samples of channel impulse responses (CIRs) at specific T-R separation distances, where the number of samples and the range of T-R separation distances are to be specified by users, as explained in the following subsection. It takes about 22 minutes to generate and save 100 CIRs and all the output files (five .png files, five sets of .txt files and five .mat files for each CIR simulation run, as well as one .png file, three sets of .txt files and three .mat files for the 100 simulation runs, as detailed in later subsections) on a PC server of Intel Xeon E5620 with two processors (2.40 $\mathrm{GHz}$ and $2.39 \mathrm{GHz}$ ) and 96.0 GB RAM [10]. NYUSIM runs about 30 times faster than our 3GPP MATLAB simulator.

\section{A. Input Parameters}

There are 28 input parameters to the simulator, which are grouped into two main categories: Channel Parameters and Antenna Properties, as shown on the GUI in Fig. 2. The panel Channel Parameters contains 16 fundamental input parameters about the propagation channel, and the panel Antenna Properties contains 12 input parameters related to the transmitter (TX) and receiver (RX) antenna arrays.

\section{B. Output Files}

1) Output Figure Files: For each simulation run, five figures will be generated and stored that are based on the particular results of the simulation that is being run, and an additional figure of path loss scatter plot will be generated and stored after $N(N \geq 1)$ continuous simulation runs with the same input parameters are complete. Regardless of the number of simulation runs (RX locations), the five figures generated from the first simulation run, as well as the last figure generated for $N$ continuous simulation runs, will pop up on the screen for visual purposes. The six output figures are: three-dimensional (3D) AOD power spectrum, 3D AOA power spectrum, a sample omnidirectional power delay profile (PDP), a sample directional PDP with strongest power, a series of PDPs over different receive antenna elements, and a path loss scatter plot. Fig. 3 illustrates a simulated AOA power spectrum, with the corresponding simulated omnidirectional PDP depicted in Fig. 4.

Fig. 5 displays the path loss scatter plot generated after $N$ continuous simulation runs, which shows omnidirectional path loss and directional path loss values for over the entire distance range generated from the $N$ continuous simulation runs, along with the fitted PLE and shadow fading standard deviation using the minimum-mean-square-error (MMSE) method [15], [17]. In the legend of Fig. 5, $n$ denotes the PLE, $\sigma$ is the shadow fading standard deviation, "omni" denotes omnidirectional, "dir" represents directional, and "dir-best" means the direction with the strongest received power. For producing the directional path loss at each RX location, NYUSIM searches for all possible pointing angles in increments of the azimuth and elevation HPBWs of the TX/RX antenna specified by the user on the GUI after first generating the omnidirectional PDP. The TX/RX antenna gain pattern is calculated by NYUSIM using Eqs. (45) and (46) in [13] based on the azimuth and elevation HPBWs of TX and RX antennas specified by the user on the GUI. The directional path loss is equal to the transmit power plus the TX and RX antenna gains, minus the directional received power [11], [12]. For generating Fig. 5. the antenna azimuth and elevation HPBWs are set to $10.9^{\circ}$ and $8.6^{\circ}$, respectively, at both the TX and the RX, to match the antenna HPBWs used in the $28 \mathrm{GHz}$ measurements [11], [12]. The simulated PLE and shadow fading standard deviation values agree well with the measured results presented in Table $\mathrm{V}$ and Table VIII of [12]. Directional path loss and directional PLE will always be larger (i.e., a directional channel is more lossy) than the omnidirectional case, because the directional antenna will spatially filter out many MPCs due to its directional pattern, such that the RX receives fewer MPCs hence less energy, thereby the directional path loss is higher after removing the antenna gain effect from the received power [12], [17].

2) Output Data Files: For each simulation run, five sets of .txt files and five corresponding .mat files are generated, namely, "AODLobePowerSpectrum $n \_L o b e x . t x t$ ", "AODLobePowerSpectrumn.mat",

"AOALobePowerSpectrumn_Lobex.txt",

"AOALobePowerSpectrumn.mat",

"OmniPDPn.mat",

"OmniPDPn.txt", "DirectionalPDPn.mat", "SmallScalePDPn.txt", and "SmallScalePDPn.mat", where $n$ denotes the $n^{\text {th }} \mathrm{RX}$ location (i.e., $n^{\text {th }}$ simulation run), and $x$ represents the $x^{t h}$ spatial lobe. After $N$ continuous simulation runs, another three .txt files and three corresponding .mat files are produced, i.e., "BasicParameters.txt", "BasicParameters.mat", "OmniPDPInfo.txt", “OmniPDPInfo.mat", "DirPDPInfo.txt", and "DirPDPInfo.mat".

The files "BasicParameters.txt" and "BasicParameters.mat" subsume all the input parameter values as shown on the GUI when running the simulation. The files "OmniPDPInfo.txt" and "OmniPDPInfo.mat" contain four columns where each column represents a key parameter for each of the $N$ omnidirectional PDPs from $N$ continuous simulation runs, including T-R separation distance, omnidirectional received power, omnidirectional path loss, and omnidirectional root-meansquare (RMS) delay spread. The files "DirPDPInfo.txt" and "DirPDPInfo.mat" contain 10 columns where each column represents a key parameter for each of the directional PDPs from $N$ continuous simulation runs, such as time delay, received power, phase, azimuth and elevation AODs and AOAs of each resolvable MPC (i.e., antenna pointing angle), along with directional path loss and directional RMS delay spread.

Each "AODLobePowerSpectrum $n$ Lobe $x$ " file is associated with a corresponding 3D AOD power spectrum output 


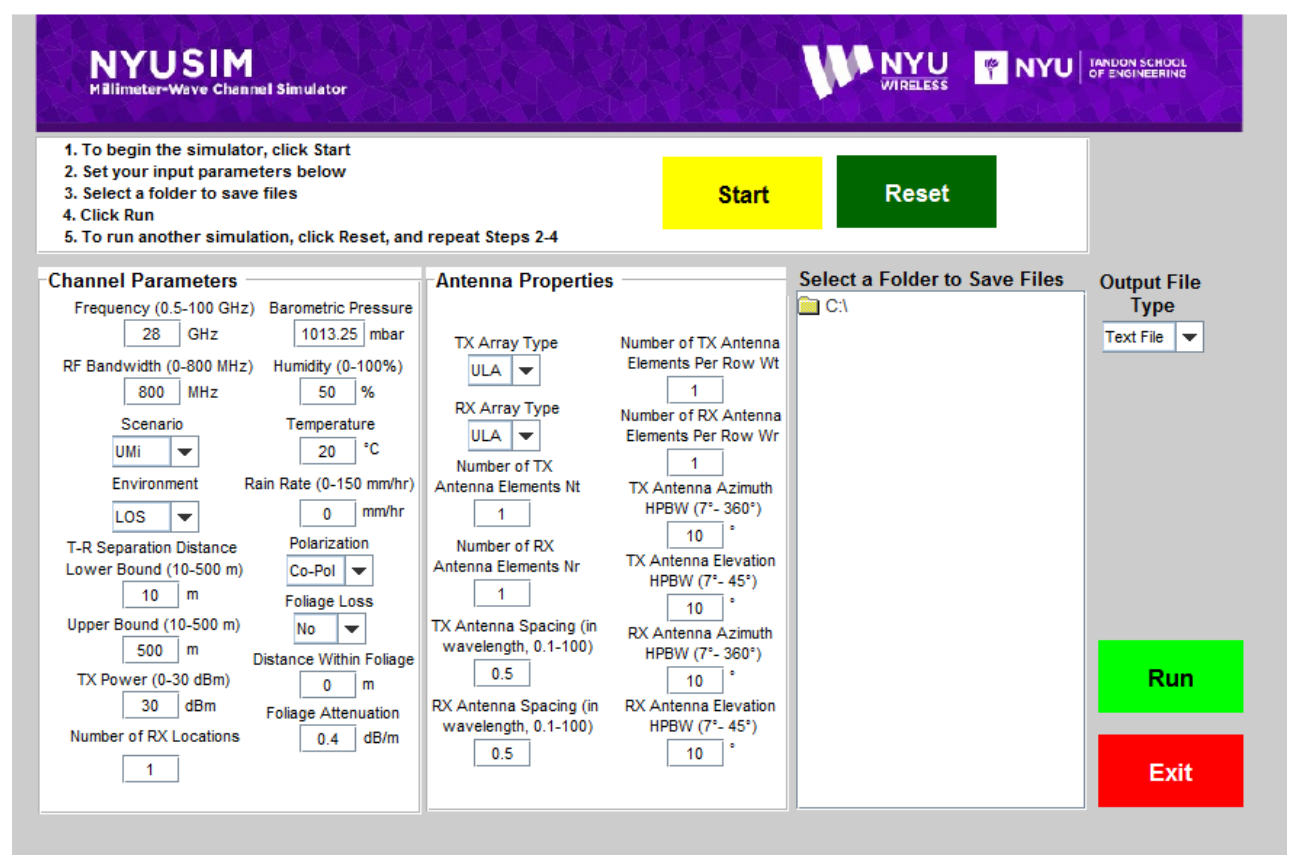

Fig. 2: Graphical User Interface (GUI) of the NYUSIM channel model simulator.

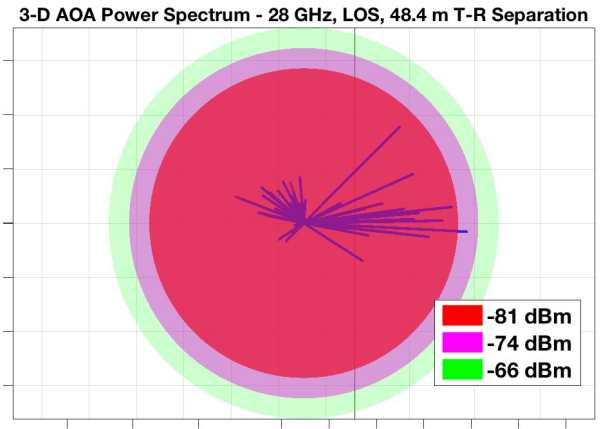

Fig. 3: Example of a simulated AOA power spectrum.

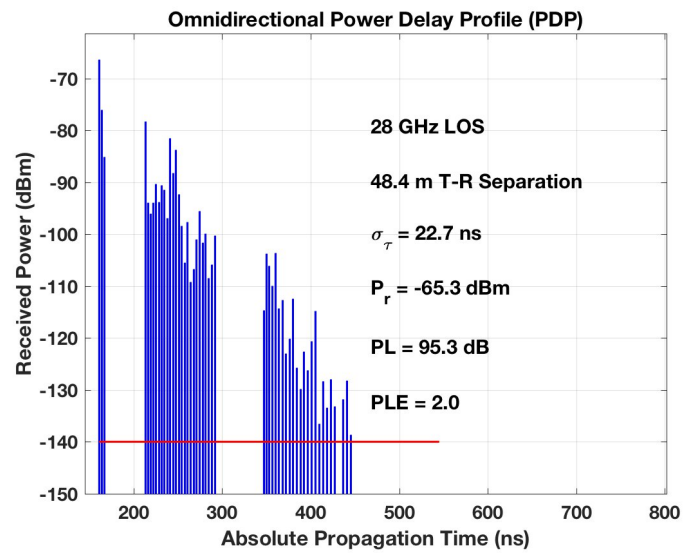

Fig. 4: Example of a simulated omnidirectional PDP.

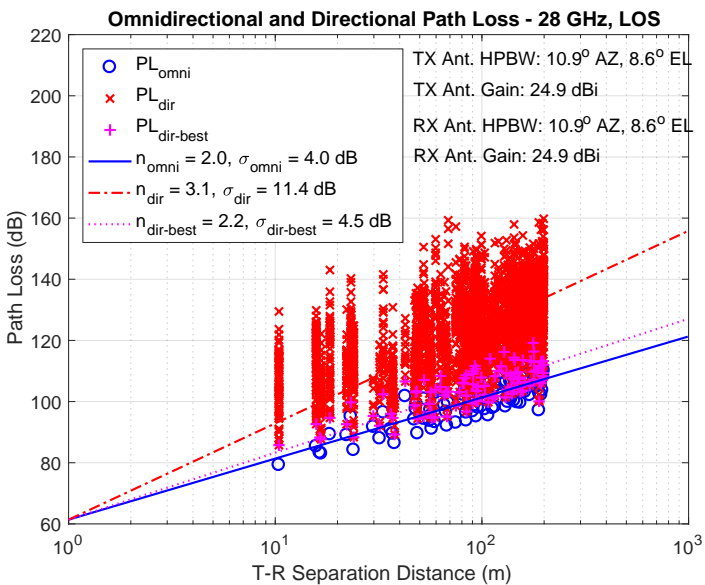

Fig. 5: Example of a scatter plot showing the omnidirectional and directional path loss values generated from NYUSIM with 100 simulation runs.

figure, and contains five parameters (columns) of each resolvable MPC in an AOD spatial lobe, namely: pathDelay (ns), pathPower (mWatts), pathPhase (rad), AOD (degree), and ZOD (degree). Similar parameters are contained in each .txt and .mat file "AOALobePowerSpectrum $n \_$Lobe $x$ " that is associated with the output figure of 3D AOA power spectrum.

Each "OmniPDP $n$ " file is associated with an omnidirectional PDP output figure, and contains two columns: the first column denotes the propagation time delay in nanoseconds, and the second column represents the received power in $\mathrm{dBm}$. Each .txt and .mat file "DirectionalPDP $n$ " is associated with the output figure of directional PDP with strongest power, and contains two columns: the first column denotes 
the propagation time delay in nanoseconds, and the second column represents the received power in $\mathrm{dBm}$. Each .txt and .mat file "SmallScalePDPn" is associated with the output figure of the series of omnidirectional PDPs over different RX antenna elements, and contains three columns: the RX antenna separation in terms of number of wavelengths, the propagation time delay in nanoseconds, and the received power in $\mathrm{dBm}$.

\section{APPLICATIONS OF NYUSIM}

The output figures and data files generated from NYUSIM can be used in various ways based on users' needs, e.g., to simulate CIRs for mmWave systems, to investigate MIMO channel performance, and to perform BER simulation [7], [33].

\section{A. MIMO Channel Condition Number}

First we show an example of how to obtain the condition number of a MIMO channel in NYUSIM by making use of the output data file "DirPDPInfo.txt" or "DirPDPInfo.mat", assuming orthogonal frequency division multiplexing (OFDM) modulation is utilized.

The condition number of a matrix is defined as the ratio of the largest to smallest singular value in the singular value decomposition of a matrix, and is a metric to characterize the quality of MIMO channels in the context of wireless communications [24], [34]-[36]. The condition number will be high (e.g., over $20 \mathrm{~dB}$ ) if the minimum singular value is close to zero, and will be $0 \mathrm{~dB}$ if singular values are equal. Physically, a small condition number value (e.g., below 20 $\mathrm{dB}$ ) indicates good orthogonality of different sub-channels (a sub-channel usually has a distinct spatial direction), and the channel gains are comparable in different spatial directions. The rank of a matrix is the dimension of the vector space generated (or spanned) by its columns (or rows) [37], and it determines how many data streams can be multiplexed over the channel in the context of MIMO communications [22], [34], [38]. The condition number is related to the rank of a matrix: the matrix has full rank (the highest rank) when the condition number is equal or close to $0 \mathrm{~dB}$ (the lowest theoretical condition number).

Now we explore the condition number of a MIMO channel matrix for a sub-carrier in an OFDM system. As described above, the output data files "DirPDPInfo.txt" and "DirPDPInfo.mat" contain paramount parameters of each resolvable MPC, which will be useful in generating the MIMO channel coefficient for an OFDM sub-carrier. Assuming uniform linear arrays (ULAs) are employed at both the TX and $\mathrm{RX}$, the equation for generating such a channel coefficient is given by (4), which is adapted from Eq.(2) in [39], where $h_{m, k}(f)$ denotes the MIMO channel coefficient between the $m^{t h}$ transmit antenna and the $k^{t h}$ receive antenna for the sub-carrier $f, p$ represents the $p^{t h}$ resolvable MPC, $\alpha$ is the amplitude of the channel gain, $\Phi$ denotes the phase of the MPC, $\tau$ represents the time delay, $d_{T}$ and $d_{R}$ are the antenna element spacing at the TX and RX, respectively, while $\phi$ and $\varphi$ denote the azimuth AOD and AOA, respectively. All of the above parameters can be extracted from the file "DirPDPInfo.txt" or "DirPDPInfo.mat". For each sub-carrier $f$ in a MIMO-OFDM system, there exists an $N_{r} \times N_{t}$ channel matrix $\mathbf{H}$ whose elements are $h_{m, k}(f)$, where $m=1, \ldots, N_{t}$ and $k=1, \ldots, N_{r}$. The condition number of $\mathbf{H}$ can be obtained consequently. The above mentioned approach along with the following input parameters settings on the NYUSIM GUI were used to run a simulation:

- Frequency: $28 \mathrm{GHz}$

- RF bandwidth: $800 \mathrm{MHz}$

- Scenario: UMi

- Environment: LOS

- Lower Bound of T-R Separation Distance: $100 \mathrm{~m}$

- Upper Bound of T-R Separation Distance: $100 \mathrm{~m}$

- TX Power: $30 \mathrm{dBm}$

- Barometric Pressure: 1013.25 mbar

- Humidity: $50 \%$

- Temperature: $20^{\circ} \mathrm{C}$

- Rain Rate: $0 \mathrm{~mm} / \mathrm{hr}$

- Polarization: Co-Pol

- Foliage Loss: No

- TX Array Type: ULA

- RX Array Type: ULA

- Number of TX Antenna Elements $N_{t}: 2$

- Number of RX Antenna Elements $N_{r}: 2$

- TX Antenna Spacing: 0.5 wavelength

- RX Antenna Spacing: 0.5 wavelength

- Number of TX Antenna Elements Per Row $W_{t}: 2$

- Number of RX Antenna Elements Per Row $W_{r}: 2$

- TX Antenna Azimuth HPBW: $10^{\circ}$

- TX Antenna Elevation HPBW: $10^{\circ}$

- RX Antenna Azimuth HPBW: $10^{\circ}$

- RX Antenna Elevation HPBW: $10^{\circ}$

For the simulation, assuming the frequency interval between adjacent sub-carriers is $10 \mathrm{MHz}$, which corresponds to $800 \mathrm{MHz} / 10 \mathrm{MHz}+1=81$ sub-carriers, we performed a simulation to obtain an intuitive observation on the MIMO channel matrix coefficients and condition number of the channel matrix. Fig. 6 illustrates the resultant magnitude of the four channel coefficients, wherein the transmitted wideband signal undergoes frequency-selective fading, and the fading magnitude varies for different TX-RX antenna element combinations.

Next, with the identical input parameters listed above, but the frequency interval between adjacent sub-carriers set to 500 $\mathrm{kHz}$, which corresponds to $800 \mathrm{MHz} / 500 \mathrm{kHz}+1=1601$ sub-carriers, we performed a simulation to emulate a random MIMO channel realization with the input parameters described above. Then the following changes are made to the four input parameters below with all the other input parameter values remaining the same:

- Number of TX Antenna Elements $N_{t}: 3$

- Number of RX Antenna Elements $N_{r}: 3$

- Number of TX Antenna Elements Per Row $W_{t}: 3$

- Number of RX Antenna Elements Per Row $W_{r}: 3$

Fig. 7 depicts the empirical cumulative distribution function (CDF) of the condition number of channel matrices for OFDM sub-carriers with the above two sets of input parameters from the simulation. It is apparent from Fig. 7 that the condition numbers of the individual OFDM sub-carriers for a $3 \times 3$ MIMO channel is about $18 \mathrm{~dB}$ larger compared to the $2 \times 2$ case on average, and the relatively large condition number of 


$$
h_{m, k}(f)=\sum_{p} \alpha_{m, k, p} e^{j \Phi_{m, k, p}} e^{-j 2 \pi f \tau_{m, k, p}} e^{-j 2 \pi d_{T} m \sin \left(\phi_{m, k, p}\right)} e^{-j 2 \pi d_{R} k \sin \left(\varphi_{m, k, p}\right)}
$$

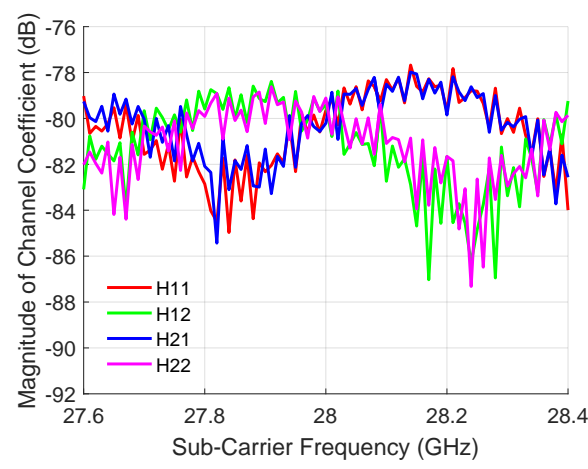

Fig. 6: MIMO channel matrix coefficient magnitudes for OFDM sub-carriers with two transmit and two receive antenna elements for MIMO-OFDM channels in one simulation run.

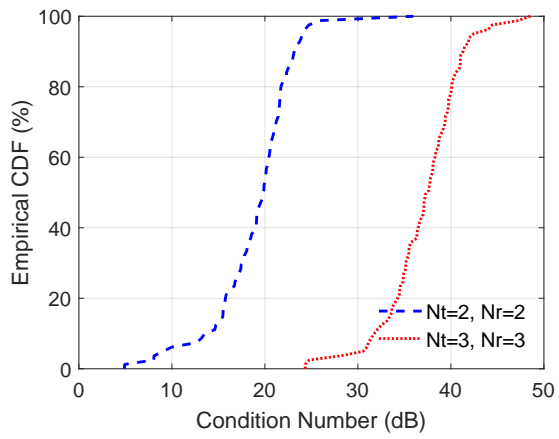

Fig. 7: CDF of the condition number of channel matrices for OFDM sub-carriers with different transmit and receive antenna elements $N_{t}$ and $N_{r}$ for MIMO-OFDM channels in one simulation run.

the $3 \times 3$ channel matrix may stem from the fact that the matrix is rank deficient.

\section{B. Spectral Efficiency Comparison Between $3 G P P$ and NYUSIM Channel Models}

As mentioned in Section II-B, the 3GPP TR 38.900 Release 14 channel model [32] contains unrealistic number of clusters (e.g., 19 clusters for UMi NLOS) and up to 20 rays per cluster, which is excessively large and not borne out by measurements. In this subsection, we use the 3GPP TR 38.900 Release 14 channel model [32] and NYUSIM channel model to analyze and compare the spectral efficiency (SE) for mmWave MIMO channels.

Let us assume a single-cell single-user MIMO system operating at $28 \mathrm{GHz}$ with an RF bandwidth of $100 \mathrm{MHz}$ in the UMi scenario. The base station is equipped with 256 antenna elements comprising a uniform rectangular array (URA), where the antenna pattern on Page 23 of [32] is adopted. The user has 16 antenna elements constituting a

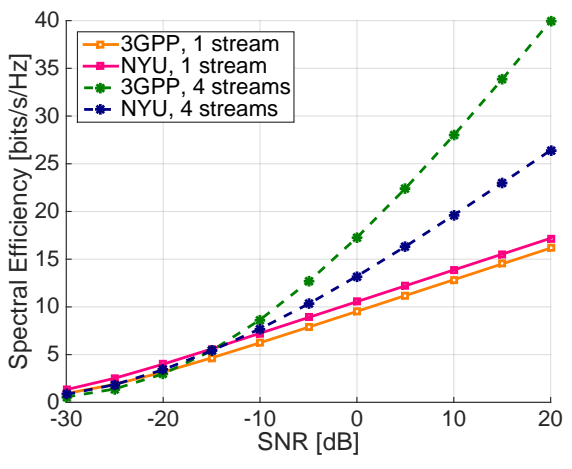

Fig. 8: Spectral efficiency achieved by hybrid beamforming for a $256 \times 16$ system at $28 \mathrm{GHz}$ with rectangular antenna arrays at the transmitter and receiver with four RF chains. The results were averaged over 200 simulation runs.

URA. Signal-to-noise ratios (SNRs) are fixed at certain values to investigate the SE achieved by the 3GPP [32] and NYUSIM channel models. Two hundred random channel realizations with distances ranging from 10 to $435 \mathrm{~m}$ were performed for each channel model.

Fig. 8 illustrates the SE achieved by the hybrid beamforming algorithm proposed in [40] for a $256 \times 16 \mathrm{mmWave}$ system at $28 \mathrm{GHz}$ for various numbers of data streams $N_{s}$, using the 3GPP and NYUSIM models. As shown by Fig. 8, the SE generated by the $3 \mathrm{GPP}$ channel model is just slightly smaller than that yielded by the NYUSIM channel model for $N_{s}=1$, but is much larger for $N_{s}=4$. For instance, for an SNR of $20 \mathrm{~dB}$ and $N_{s}=4$, the $3 \mathrm{GPP}$ SE (40 bits/s/Hz) is about 13 bits/s/Hz greater than the NYUSIM SE (about $27 \mathrm{bits} / \mathrm{s} / \mathrm{Hz}$ ). This is because NYUSIM yields one or two strong dominant clusters and much weaker non-dominant clusters, while the 3GPP model has less focused directional energy than what realistically exists. The results indicate that the $3 \mathrm{GPP}$ channel model is optimistic when predicting diversity and the achievable SE at mmWave frequencies, while NYUSIM provides realistic channel parameters and SE predictions due to the use of extensive real-world measurement data at mmWave frequencies. NYUSIM can help avoid system errors inherent with legacy modeling approaches.

\section{CONCLUSion}

This paper has presented an open-source channel software simulator, NYUSIM, developed from extensive broadband propagation measurements at mmWave frequencies. NYUSIM recreates wideband PDPs/CIRs and channel statistics for a variety of carrier frequencies, RF bandwidths, antenna beamwidths, environment scenarios, and atmospheric conditions, and is equipped with a GUI that makes the simulator more user-friendly. Over 7000 downloads have already been logged by major corporations and universities worldwide. Simulated results from NYUSIM match well with the measured data. Application examples study channel conditions 
and compare MIMO channel performance between 3GPP and NYUSIM models. NYUSIM can be employed to perform various other types of analysis and is useful for $5 \mathrm{G}$ communication system development and deployment.

\section{REFERENCES}

[1] S. Y. Seidel, K. Takamizawa, and T. S. Rappaport, "Application of second-order statistics for an indoor radio channel model," in IEEE 39th Vehicular Technology Conference, May 1989, pp. 888-892 vol.2.

[2] S. Jaeckel et al., "QuaDRiGa: A 3-D multi-cell channel model with time evolution for enabling virtual field trials," IEEE Transactions on Antennas and Propagation, vol. 62, no. 6, pp. 3242-3256, June 2014.

[3] Y. Yu et al., "Propagation model and channel simulator under indoor stair environment for machine-to-machine applications," in 2015 AsiaPacific Microwave Conference, vol. 2, Dec. 2015, pp. 1-3.

[4] T. S. Rappaport et al., "Statistical channel impulse response models for factory and open plan building radio communicate system design," IEEE Transactions on Communications, vol. 39, no. 5, pp. 794-807, May 1991.

[5] Wireless Valley Communications, Inc., SMRCIM Plus 4.0 (Simulation of Mobile Radio Channel Impulse Response Models) Users Manual, Aug. 1999.

[6] V. K. Rajendran et al., "Concepts and implementation of a semantic web archiving and simulation system for rf propagation measurements," in 2011 IEEE Vehicular Technology Conference (VTC Fall), Sept 2011, pp. 1-5.

[7] V. Fung et al., "Bit error simulation for pi/4 DQPSK mobile radio communications using two-ray and measurement-based impulse response models," IEEE Journal on Selected Areas in Communications, vol. 11, no. 3, pp. 393-405, Apr. 1993.

[8] J. I. Smith, "A computer generated multipath fading simulation for mobile radio," IEEE Transactions on Vehicular Technology, vol. 24, no. 3, pp. 39-40, Aug 1975.

[9] R. H. Clarke, "A statistical theory of mobile-radio reception," The Bell System Technical Journal, vol. 47, no. 6, pp. 957-1000, July 1968.

[10] New York University, NYUSIM, 2016.2 [Online]. Available: http://wireless.engineering.nyu.edu/ 5gmillimeter-wave-channel-modeling-software/.

[11] T. S. Rappaport et al., "Millimeter wave mobile communications for 5G cellular: It will work!" IEEE Access, vol. 1, pp. 335-349, 2013.

[12] _ "Wideband millimeter-wave propagation measurements and channel models for future wireless communication system design (Invited Paper)," IEEE Transactions on Communications, vol. 63, no. 9, pp. 3029-3056, Sep. 2015.

[13] M. K. Samimi and T. S. Rappaport, "3-D millimeter-wave statistical channel model for 5G wireless system design," IEEE Transactions on Microwave Theory and Techniques, vol. 64, no. 7, pp. 2207-2225, July 2016.

[14] _ _ "Local multipath model parameters for generating 5G millimeterwave 3GPP-like channel impulse response," the 10th European Conference on Antennas and Propagation (EuCAP 2016), April 2016.

[15] S. Sun et al., "Investigation of prediction accuracy, sensitivity, and parameter stability of large-scale propagation path loss models for $5 \mathrm{G}$ wireless communications," IEEE Transactions on Vehicular Technology, vol. 65, no. 5, pp. 2843-2860, May 2016.

[16] _ _ "Synthesizing omnidirectional antenna patterns, received power and path loss from directional antennas for $5 \mathrm{G}$ millimeter-wave communications," in 2015 IEEE Global Communications Conference (GLOBECOM), Dec. 2015, pp. 1-7.

[17] G. R. MacCartney, Jr. et al., "Indoor office wideband millimeter-wave propagation measurements and channel models at 28 and $73 \mathrm{GHz}$ for ultra-dense 5G wireless networks," IEEE Access, vol. 3, pp. 2388-2424, Oct. 2015.

[18] _ - "Millimeter wave wireless communications: New results for rural connectivity," in All Things Cellular16, in conjunction with ACM MobiCom, Oct. 2016.
[19] G. R. MacCartney, Jr. and T. S. Rappaport, "Study on 3GPP rural macrocell path loss models for millimeter wave wireless communications," in 2017 IEEE International Conference on Communications (ICC), May 2017, pp. 1-7.

[20] MathWorks. [Online]. Available: https://www.mathworks.com/.

[21] R. B. Ertel et al., "Overview of spatial channel models for antenna array communication systems," IEEE Personal Communications, vol. 5, no. 1, pp. 10-22, Feb 1998.

[22] S. Sun et al., "MIMO for millimeter-wave wireless communications: beamforming, spatial multiplexing, or both?" IEEE Communications Magazine, vol. 52, no. 12, pp. 110-121, Dec. 2014.

[23] H. J. Liebe et al., "Propagation modeling of moist air and suspended water/ice particles at frequencies below $1000 \mathrm{GHz}$," AGARD Conference Proceedings 542, May 1993.

[24] T. S. Rappaport, R. W. Heath, Jr., R. C. Daniels, and J. N. Murdock, Millimeter Wave Wireless Communications. Pearson/Prentice Hall 2015.

[25] K. Bullington, "Radio propagation at frequencies above 30 megacycles," Proceedings of the IRE, vol. 35, no. 10, pp. 1122-1136, Oct. 1947.

[26] H. T. Friis, "A note on a simple transmission formula," Proceedings of the IRE, vol. 34, no. 5, pp. 254-256, May 1946.

[27] M. Hata, "Empirical formula for propagation loss in land mobile radio services," IEEE Transactions on Vehicular Technology, vol. 29, no. 3, pp. 317-325, Aug 1980.

[28] J. B. Andersen, T. S. Rappaport, and S. Yoshida, "Propagation measurements and models for wireless communications channels," IEEE Communications Magazine, vol. 33, no. 1, pp. 42-49, Jan 1995.

[29] "Investigation of prediction accuracy, sensitivity, and parameter stability of large-scale propagation path loss models from $500 \mathrm{MHz}$ to 100 GHz." [Online]. Available: http://wireless.engineering.nyu.edu/presentations/ NTIA-propagation-presentation-JUNE-15-2016_v1-3.pdf

[30] P. Kyosti et al., "WINNER II channel models," European Commission, IST-WINNER, Tech. Rep. D1.1.2.

[31] 3GPP, "Technical specification group radio access network; study on 3d channel model for lte (release 12)," 3rd Generation Partnership Project (3GPP), TR 36.873 V12.2.0, June 2015. [Online]. Available: http://www.3gpp.org/dynareport/36873.htm

[32] - "Study on channel model for frequency spectrum above $6 \mathrm{GHz}$," 3rd Generation Partnership Project (3GPP), TR 38.900 V14.2.0, Dec. 2016. [Online]. Available: http://www.3gpp.org/DynaReport/38900.htm

[33] B. Thoma et al., "Simulation of bit error performance and outage probability of pi/4 DQPSK in frequency-selective indoor radio channels using a measurement-based channel model," in IEEE Global Telecommunications Conference (GLOBECOM), Dec 1992, pp. 1825-1829 vol.3.

[34] Q. H. Abbasi et al., "Condition number variability of ultra wideband MIMO on body channels," in 2016 International Workshop on Antenna Technology (iWAT), Feb 2016, pp. 167-169.

[35] R. W. Heath and D. J. Love, "Multimode antenna selection for spatial multiplexing systems with linear receivers," IEEE Transactions on Signal Processing, vol. 53, no. 8, pp. 3042-3056, Aug 2005.

[36] X. Lu et al., "An improved semi-orthogonal user selection algorithm based on condition number for multiuser MIMO systems," China Communications, vol. 11, no. 13, pp. 23-30, Supplement 2014.

[37] N. Bourbaki, Elements of Mathematics, Algebra I. Hermann 1974.

[38] M. Matthaiou et al., "Reduced complexity detection for ricean MIMO channels based on condition number thresholding," in 2008 International Wireless Communications and Mobile Computing Conference, Aug 2008, pp. 988-993.

[39] A. Adhikary et al., "Joint spatial division and multiplexing for mmwave channels," IEEE Journal on Selected Areas in Communications, vol. 32, no. 6, pp. 1239-1255, June 2014.

[40] O. E. Ayach et al., "Spatially sparse precoding in millimeter wave MIMO systems," IEEE Transactions on Wireless Communications, vol. 13, no. 3, pp. 1499-1513, March 2014. 